\title{
Pancreatic cystic lesions in diabetes mellitus patients
}

\author{
Martyna Rozek ${ }^{1}$, Michal Lipinski ${ }^{1}$, Ewa Jozefik ${ }^{1}$, Zuzanna Znajdek ${ }^{1}$, Marta Kiziak ${ }^{1}$, Marta Sznurkowska ${ }^{1}$, \\ Jacek Tatur ${ }^{1}$, Malgorzata Degowska1, Grazyna Rydzewska ${ }^{1,2}$
}

${ }^{1}$ Department of Gastroenterology, Central Clinical Hospital of the Ministry of Interior, Warsaw, Poland

${ }^{2}$ Collegium Medicum, Jan Kochanowski University, Kielce, Poland

Gastroenterology Rev 2021; 16 (1): 62-66

DOI: https://doi.org/10.5114/pg.2020.96080

Key words: diabetes mellitus, pancreatic pseudocyst, endosonography.

Address for correspondence: Martyna Rozek, Department of Gastroenterology, Central Clinical Hospital of the Ministry of Interior, Warsaw, Poland, phone: +48 600153 401, e-mail: martyna.rozek3@gmail.com

\begin{abstract}
Introduction: According to the literature exocrine pancreatic insufficiency is relatively common among patients with diabetes mellitus (DM). Pseudocysts are the most common cystic lesions and may be formed in the setting of acute or chronic pancreatitis. However, whether DM is involved or not in pancreatic cyst formation is still not well established.

Aim: To investigate the frequency and risk factors of cystic lesions in diabetic patients.

Material and methods: One hundred and sixty-one patients with DM, with no previous history of pancreatic diseases, were prospectively included in the study. Endosonography followed by fine needle aspiration biopsy was then performed.

Results: Finally, 33 of 161 patients (20.5\%) were recognized with cystic lesions of the pancreas. Among them 5 patients were classified as cystic neoplasms, and 28 as pseudocysts. In the group of patients with pseudocysts, cystic lesions were significantly more prevalent in individuals with DM lasting less than 3 years. Prevalence of cystic lesions was significantly higher in metformin users in comparison to other diabetic patients $(p<0.05)$. Cystic lesions were more frequent in patients above 50 years of age $(p<0.05)$.

Conclusions: The prevalence of cystic lesions in the diabetic population is higher than in the general population. DM seems to play a major role in the process of cyst development, especially in patients without previous history of pancreatitis. Higher prevalence of cystic lesions in early diabetes seems to be the first stage of pancreatic injury. The exact role of diabetes duration and type of treatment should be established.
\end{abstract}

\section{Introduction}

In recent years, there have been reports regarding the problem of risk of accompanying pancreatic diseases of different nature in patients with diagnosed diabetes [1-4]. However, the influence of diabetes on the possibility of pancreatic lesions, which hypothetically may have cancer potential, is still not fully known. The pathophysiology of this issue is quite complex. Studies carried out in recent years in the population of diabetic patients provide evidence for consequences that are not only morphological changes in the organ itself, but also disorders of exocrine function, which in recent years have been called diabetic exocrine pancreatopathy $[5,6]$.

At present, thanks to the progress made in medical imaging, an upward trend in the detection of focal changes in the pancreas is observed [7]. It is also associated with a certain chance to detect potential neoplastic lesions at the earliest possible stage - which could affect the patient's prognosis. In recent years, endoscopic ultrasonography (EUS) has been considered the most sensitive method to detect even small focal pancreatic lesions [8]. Additionally, EUS enables the collection of material for analysis (histological, cytological or biochemical) of suspected lesions. The results obtained determine the further management of the patient $[9,10]$.

It is estimated that the occurrence of cystic changes in the pancreas detected in imaging findings based on different studies in the general population is from $1.2 \%$ to $19 \%[10,11]$. To date, only a few epidemiological studies have been published which aimed to determine the frequency of cystic lesions in the pancreas in patients with diabetes and the incidence of diabetes in patients with cystic lesions in the pancreas [11].

The studies published so far also do not indicate a specific factor responsible for the formation of these 
lesions in the population of people with diabetes. However, there is an increased presence of cystic lesions in the elderly, with high body mass index (BMI) and a history of diabetes mellitus (DM) $[11,12]$.

Even accidentally detected single pancreatic cysts may constitute changes with potential cancer growth and be associated with an increased risk of cancer in patients under 65 [13-15]. In addition, most studies indicate an increased risk of pancreatic cancer in patients with diagnosed diabetes, especially type $2[16,17]$.

\section{Aim}

The aim of the study was to determine the frequency and nature of pancreatic focal lesions occurring in patients with diabetes, taking into consideration potential risk factors.

\section{Material and methods}

The research was carried out in the Department of Gastrology of the Central Clinical Hospital of the Ministry of Interior in Warsaw. 161 patients with already diagnosed diabetes mellitus type 2 were included in the prospective study. Every individual qualified for the study had EUS examination. Particular focus was on potential presence of pancreatic lesions, both cystic and solid. In selected cases, due to indications, endoscopic biopsy was performed. Endosonography was performed using Pentax and Fujinon instruments. All examinations were conducted by two expert endoscopists. 76 women (mean age: 63) and 85 men (mean age: 61 ) belonged to the research group. The youngest patient was 20 and the oldest 88 years old (Table I). In addition, the literature reports a noticeably higher incidence of pancreatic lesions in patients over 50 years of age; therefore we chose this age limit to study the incidence of lesions, proving it.

None of them had previous disorders of the pancreas in medical history. The exclusion criterion was chronic pancreatitis, diagnosed while conducting the study. Age and time of diabetes mellitus were included in the data analysis. Control subjects were investigated with regard to the time of the disease - lasting less than 36 months, or lasting more than 36 months. The analysis, which aimed to define the influence of the application of the particular treatment specific to diabetes (metformin, insulin, diet) on the risk of occurrence of focal lesions in the pancreas, was conducted in the examined group.

\section{Results}

In the examined group of 161 patients, 33 patients were found to have at least one cystic lesion of the pancreas $(20.5 \%)$. Based on detailed analysis of the material, 5 (3.1\%) cystic neoplasms and 28 (17.4\%) pseudocysts were differentiated from 33 patients (Table II).

We did not confirm a statistically significant difference in the occurrence of pseudocysts in groups of patients with short-term and long-term diabetes (16 vs. 12 patients; $p>0.05$ ) (Table III).

The calculations showed a statistically significant difference $(p<0.05)$ in relation to the occurrence of pseudocysts in metformin-using or non-using individuals. The frequency of these changes was significantly

Table I. Demographics

\begin{tabular}{lc} 
Population of patients & $\boldsymbol{N}(\%)$ \\
\hline \begin{tabular}{l} 
Gender: \\
\hline Female
\end{tabular} & $76(47.2)$ \\
\hline Male & $85(52.8)$ \\
\hline \begin{tabular}{l} 
Age: \\
\hline Mean
\end{tabular} & $25(15.5)$ \\
\hline <0 years old & $136(84.5)$ \\
\hline$\geq 50$ years old & $41(25.5)$ \\
\hline Diabetes: & $48(29.8)$ \\
\hline \begin{tabular}{l} 
Short-term \\
\hline Long-term
\end{tabular}
\end{tabular}

Table II. Type of cystic lesions in the studied population and age of patients with cystic lesions $(n=161)$

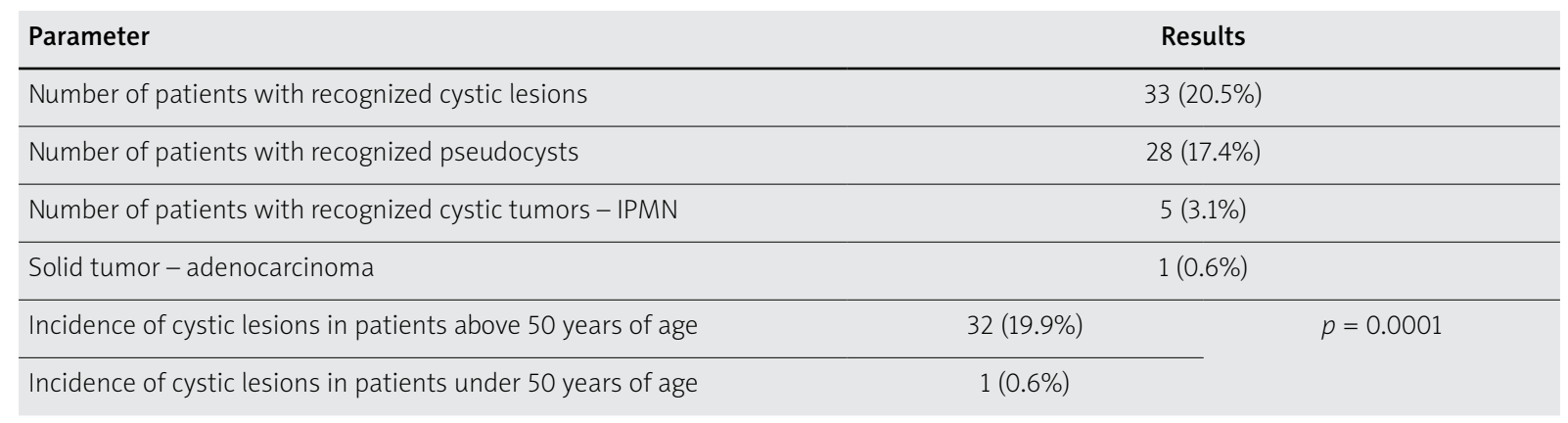


Table III. Time from diagnosis of diabetes and incidence of pseudocysts

\begin{tabular}{lcc} 
Parameter & DM lasting less than $\mathbf{3 6}$ months & DM lasting more than $\mathbf{3 6}$ months \\
\hline Number of patients with recognized pseudocysts & 16 patients & 12 patients \\
\hline$P$-value & $>0.05$
\end{tabular}

Table IV. Prevalence of pseudocysts in the group of persons treated with metformin and in the group of persons not treated with metformin

\begin{tabular}{lcc} 
Type of treatment & Metformin users & Non-metformin users \\
\hline Number of patients with recognized pseudocysts & 20 patients & 8 patients \\
\hline$P$-value & $<0.05$
\end{tabular}

higher in metformin users $(71.4 \%)$ compared to the non-users (28.6\%) (Table IV).

We observed a significantly higher incidence of cystic lesions in patients above 50 years of age $(p=0.0001)$.

Five lesions (3.1\% of the studied population) of intraductal papillary mucinous neoplasia (IPMN) were diagnosed. Each case of IPMN was confirmed by endoscopic biopsy with subsequent analysis of the collected material (histopathological, cytological and biochemical).

Among patients only one solid lesion was diagnosed, which was $0.6 \%$ of the studied population. After endoscopic biopsy it was diagnosed as adenocarcinoma G2.

\section{Discussion}

In the last decade there have been reports that new onset diabetes can be a prediction factor up to 12 months ahead of developing pancreatic cancer. The concept of new onset diabetes is understood differently and different time criteria (from 12 months to 36 months) are adopted [18, 19]. In our study based on reports from Illés et al. we assumed the time of 36 months from the moment of diagnosis of diabetes mellitus - as a period that may include the range in which it is possible to detect early lesions with potential risk of malignant transformation [20].

In the course of our study, our attention was particularly focused on the detection of solid lesions in patients with new onset diagnosed diabetes. However, we noted all changes (solid and cystic), and in selected cases we determined their character in endoscopic biopsy.

In our material we found only one solid lesion, which we recognized from the biopsy as adenocarcinoma G2.

What was surprising for us was the high frequency of cystic lesions, which may depend on the diagnosis available among them: 28 (17.4\%) pseudocysts and $5(3.1 \%)$ cystic tumors. Based on the endoscopic biopsy performed, in each of the 5 cases of suspected cystic tumors IPMN was diagnosed, considering biochemical, cytological and histological analysis.

Referring to the results obtained in our study, the incidence of cystic lesions in the examined population was significantly higher in patients over 50 years old. This observation is consistent with a study, published in 2019, on a large population (10 987) of patients who underwent magnetic resonanace imaging (MRI) for reasons other than pancreatic disease [21]. In this research, similarly to ours, a strong relationship between the incidence of cystic lesions in the pancreas and the age of patients was observed. What is worth mentioning is the significantly smaller percentage of cystic lesions in the pancreas found in MRI in the studied population, reaching $1.93 \%$ compared to the population studied in our research, where this percentage was $20.5 \%$ in total. Comparison of "increased risk cystic lesions" defined by Sun et al. seems interesting as in our understanding it could be interpreted as the presence of cystic tumor. In the study conducted on the Asian population [21] the percentage of high risk cystic lesions was described as $0.12 \%$, while in the group of patients in our analysis it was $3.1 \%$.

Regarding the differences between populations, the methodology of both diagnostic imaging (EUS vs. $M R I$ ) and the qualification of the patients (in our study they were patients only with diabetes) it should be considered whether the fact of diagnosed diabetes is not essential in explaining the significantly higher percentage of patients with cystic lesions in the pancreas in the group we studied. Conceivably this may explain the recently recognized condition called "diabetic exocrine pancreatopathy" which is associated with various non-specific morphological changes in the pancreas which require further testing and defining specific diagnostic criteria. 
Diabetes mellitus is associated with increased risk of tumors, particularly of the gastrointestinal tract [22]. It is noteworthy that in $71.4 \%$ of patients using metformin in our study cystic lesions were detected. Metformin administered to diabetic patients in many scientific publications has been presented as a chemopreventive factor of cancer in the pancreas, colon or liver [23, 24]. However, in recent years there have been studies showing that metformin does not reduce the risk of gastrointestinal cancers [25]. Analyzing data from observations of over 37,000 people treated with metformin, the results prove that the use of metformin does not reduce the risk of gastrointestinal cancer. Moreover, a higher risk of pancreatic cancer was observed in the group of patients using metformin with insulin and in women using metformin [25].

The results of our study revealing an increased risk of pseudocysts in people taking metformin pose further questions about the influence of this drug on the development of various types of lesions (proinflammatory and potentially cancerous) in the pancreas.

Our analysis is the next one suggesting that diabetes seems to play an overriding role in the development of cystic lesions in the pancreas, which may become part of the recently described disease unit called diabetic pancreatopathy [26]. This should not, of course, relieve us from oncological vigilance in the case of cystic lesions (especially cystic tumors) in patients with diabetes and other high-risk factors.

Early detection of both cystic and solid lesions by means of EUS and FNA-EUS (endoscopic ultrasonography-guided fine-needle aspiration), in patients with new-onset diabetes, may be a first diagnostic stage of these clinically unpredictable findings. Thorough selection and identification of patients with risk factors for developing pancreatic neoplasms gives rise to the creation of the most precise screening model, which would identify early stages of cystic and solid pancreatic tumors.

Research evidence in the area of cystic lesions in patients with diabetes is scarce. Our study raises both this topic and the issue of types of lesions encountered. What is more, a strong emphasis was put on the occurrence of cystic lesions, the manner of which was preceded only by a handful of scientific papers.

Noteworthy is also the carefully selected research group, which consisted of patients with diabetes, with no medical history of pancreatic disease, all of whom underwent EUS and FNA-EUS.

\section{Conclusions}

Cystic lesions in the pancreas in patients with diabetes occur more frequently than in the general popu- lation. Diabetes itself can play a major role in the pathomechanism of cyst formation, particularly in cases of patients with no prior pancreatic disorders. The development of cystic lesions in diabetic patients may be the first stage of the broadly understood process called diabetic pancreatopathy. Further research is essential to determine the frequency of focal cystic and solid lesions within the pancreas in patients with diabetes and the potential impact of drugs on their development.

\section{Conflict of interest}

The authors declare no conflict of interest.

\section{References}

1. Khadka R, Tian W, Hao X, Koirala R. Risk factor, early diagnosis and overall survival on outcome of association between pancreatic cancer and diabetes mellitus: changes and advances, a review. Int J Surg 2018; 52: 342-6.

2. Matsuda Y. Age-related pathological changes in the pancreas. Front Biosci 2018; 10: 137-42.

3. Meisterfeld R, Ehehalt F, Saeger HD, Solimena M. Pancreatic disorders and diabetes mellitus. Exp Clin Endocrinol Diabetes 2008; 116 Suppl 1: S7-12.

4. Camara SN, Yin T, Yang M, et al. High risk factors of pancreatic carcinoma. J Huazhong Univ Sci Technolog Med Sci 2016; 36: 295-304.

5. Mohapatra S, Majumder S, Smyrk TC, et al. Diabetes mellitus is associated with an exocrine pancreatopathy: conclusions from a review of literature. Pancreas 2016; 45: 1104-10.

6. Lindkvist B, Nilsson C, Kvarnström M, Oscarsson J. Importance of pancreatic exocrine dysfunction in patients with type 2 diabetes: a randomized crossover study. Pancreatology 2018; S1424-3903(18)30589-1. doi:10.1016/j.pan.2018.05.483

7. Brugge WR. Diagnosis and management of cystic lesions of the pancreas. J Gastrointest Oncol 2015; 6: 375-88.

8. Brugge WR. The use of EUS to diagnose cystic neoplasms of the pancreas. Gastrointest Endosc 2009; 69 (2 Suppl): S203-9.

9. Moparty B, Bhutani MS. The role of endoscopic ultrasound in cystic pancreatic tumor treatment. Gastroenterol Hepatol 2006; 2: 578-83.

10. Hernandez LV, Mishra G, Forsmark C, et al. Role of endoscopic ultrasound (EUS) and EUS-guided fine needle aspiration in the diagnosis and treatment of cystic lesions of the pancreas. Pancreas 2002; 25: 222-8.

11. Mizuno S, Isayama H, Nakai Y, et al. Prevalence of pancreatic cystic lesions is associated with diabetes mellitus and obesity: an analysis of 5296 individuals who underwent a preventive medical examination. Pancreas 2017; 46: 801-5.

12. Larsson SC, Permert J, Håkansson N, et al. Overall obesity, abdominal adiposity, diabetes and cigarette smoking in relation to the risk of pancreatic cancer in two Swedish population-based cohorts. Br J Cancer 2005; 93: 1310-5.

13. Chernyak V, Flusberg M, Haramati LB, et al. Incidental pancreatic cystic lesions: is there a relationship with the development of pancreatic adenocarcinoma and all-cause mortality? Radiology 2015; 274: 161-9. 
14. Tanaka S, Nakao M, loka T, et al. Slight dilatation of the main pancreatic duct and presence of pancreatic cysts as predictive signs of pancreatic cancer: a prospective study. Radiology 2010; 254: 965-72.

15. Tada M, Kawabe T, Arizumi M, et al. Pancreatic cancer in patients with pancreatic cystic lesions: a prospective study in 197 patients. Clin Gastroenterol Hepatol 2006; 4: 1265-70.

16. Szablewski L. Diabetes mellitus: influences on cancer risk. Diabetes Metab Res Rev 2014; 30: 543-53.

17. Salvatore T, Marfella R, Rizzo MR, et al. Pancreatic cancer and diabetes: a two-way relationship in the perspective of diabetologist. Int J Surg 2015; 21 Suppl 1: S72-7.

18. Tanțău A, Negrean V, Alexescu T, et al. Two different types of diabetes mellitus in pancreatic cancer population. Comparative study between new onset and long standing diabetes mellitus on 76 patients with pancreatic cancer. Rom J Intern Med 2014; 52: 18-23.

19. Sharma A, Kandlakunta H, Nagpal SJS, et al. Model to determine risk of pancreatic cancer in patients with new-onset diabetes. Gastroenterology 2018; 155: 730-9.e3.

20. Illés D, Terzin V, Holzinger G, et al. New-onset type 2 diabetes mellitus: a high-risk group suitable for the screening of pancreatic cancer? Pancreatology 2016; 16: 266-71.

21. Sun L, Wang Y, Jiang F, et al. Prevalence of pancreatic cystic lesions detected by magnetic resonance imaging in the Chinese population. J Gastroenterol Hepatol 2019; 34: 1656-62.

22. Pollak MN. Investigating metformin for cancer prevention and treatment: the end of the beginning. Cancer Discov 2012; 2 778-90.

23. Evans JM, Donnelly LA, Emslie-Smith AM, et al. Metformin and reduced risk of cancer in diabetic patients. BMJ 2005; 330 1304-5.

24. Hsieh MC, Lee TC, Cheng SM, et al The influence of type 2 diabetes and glucose-lowering therapies on cancer risk in the Taiwanese. Exp Diabetes Res 2012; 2012: 413782.

25. de Jong RG, Burden AM, de Kort $S$, et al. No decreased risk of gastrointestinal cancers in users of metformin in the Netherlands: a time-varying analysis of metformin exposure. Cancer Prev Res 2017; 10: 290-7.

26. Valente R, Waldthaler A, Scandavini CM, et al. Conservative treatment of chronic pancreatitis: a practical approach. Scand J Surg 2020; 109: 59-68.

Received: 28.04 .2020

Accepted: 5.05 .2020 